\title{
« Indiens » ou « Brésiliens »? Mobilités karipuna vers Cayenne (Guyane française)
}

"Indians" or "Brazilians"? Karipuna Mobilities towards Cayenne (French Guiana)

¿«Indios» 0 «Brasileños»? Movilidades Karipuna a Cayenne (Guayana Francesa)

\section{Gérard Collomb}

\section{(2) OpenEdition}

Journals

Édition électronique

URL : https://journals.openedition.org/remi/6312

DOI : $10.4000 /$ remi.6312

ISSN : $1777-5418$

Éditeur

Université de Poitiers

Édition imprimée

Date de publication : 1 mars 2013

Pagination : 113-131

ISBN : 979-10-90426-07-8

ISSN : 0765-0752

Référence électronique

Gérard Collomb, « «Indiens » ou « Brésiliens » ? Mobilités karipuna vers Cayenne (Guyane française) », Revue européenne des migrations internationales [En ligne], vol. $29-n^{\circ} 1$ | 2013, mis en ligne le 01 mars 2016, consulté le 14 avril 2022. URL : http://journals.openedition.org/remi/6312 ; DOI : https://doi.org/ 10.4000/remi.6312 


\section{"Indiens " ou " Brésiliens "? Mobilités karipuna vers Cayenne (Guyane française)}

\section{Gérard Collomb ${ }^{1}$}

\section{Introduction ${ }^{2}$}

La Guyane française, département depuis 1946, se situe entre le Surinam et le Brésil dans le bassin amazonien, au sein duquel circulent des flux démographiques, économiques, culturels, qui traversent et dépassent les frontières héritées de I'histoire coloniale (Piantoni, 2009a). Ces mouvements dessinent des configurations culturelles, politiques, économiques élargies, qui ouvrent sur de nouvelles territorialités et tissent de nouveaux liens entre la localité et les populations (Glick Schiller et al., 1992 ; Portes, 1999 ; Abélès, 2008). À cet égard, et par-delà des réalités institutionnelles et économiques qui les distinguent, Guyane française, Surinam et Brésil " forment système " : d'une part, l'économie de l'orpaillage pousse des milliers de garimpeiros brésiliens ${ }^{3}$ vers la Guyane et le Surinam, d'autre part, la Guyane française apparaît comme un petit bout d'un Nord politique et économique fortement attractif, enchâssé dans un Sud dont relèveraient, notamment, le Surinam et les États brésiliens voisins l'Amapá et le Pará (Piantoni, 2009a).

Parmi les populations qui participent aujourd'hui à ces mobilités sur le territoire de la Guyane, quelques groupes amérindiens s'inscrivent d'une manière particulière dans ce dispositif. Ces populations ont pratiqué de longue date des déplacements de part et d'autre de la frontière politique, qui se sont prolongés jusqu'à aujourd'hui, à travers une capacité à jouer, tout à fait consciemment et rationnellement, sur le différentiel économique, social, institutionnel qui existe

\footnotetext{
1 Chargé de recherche, IIAC/LAIOS, EHESS-CNRS, 190 avenue de France, 75013 Paris ; collomb@msh-paris.fr

2 L'enquête de terrain a été menée dans le cadre du programme ANR/Suds

"Dynamiques des circulations migratoires et mobilités transfrontalières entre Guyane, Surinam, Brésil, Guyana et Haïti " conduit entre 2008 et 2010 par l'IRD et coordonné par Luc Cambrézy. Elle a également bénéficié de l'appui de I'Observatoire Hommes-Milieux/ Oyapock (CNRS/Guyane), ainsi que de I'aide précieuse de Max Dos Santos, à la Bp.134 et à Manga.

3 L'estimation de cette population de garimpeiros, illégale, est par nature difficile, mais la gendarmerie en Guyane donne une fourchette de 5000 à 10000 personnes. Cf. Beaulieu Laure (2012) Chercheurs d'or illégaux en Guyane : le casse-tête des autorités françaises, Le Monde, 13/07/2012, [en ligne]. URL : http://www.lemonde.fr
} 
entre la Guyane et le Surinam, et entre la Guyane et le Brésil. De tels comportements ne sont nullement des stratégies récentes, qui seraient liées au grand développement contemporain des processus migratoires ; ce jeu sur la frontière est ancien, et il s'est inscrit dans une culture de la mobilité transfrontalière que ces sociétés ont reproduite tout au long de I'histoire coloniale.

L'exemple des Amérindiens karipuna, établis dans le Brésil frontalier et venant travailler en Guyane, illustre la place singulière occupée par ces groupes dans l'espace régional, mais aussi les profondes transformations que ces systèmes de mobilités ont connues lorsque s'est imposée, récemment, une frontière restée jusqu'alors virtuelle. Ces populations sont alors passées du statut d'Indiens véritablement " trans-frontaliers ", bénéficiant de fait de privilèges d'accès au territoire français, à celui de " migrants " franchissant une frontière, auxquels l'État applique des règles renforcées de limitation des mobilités.

\section{Un monde de l'Oyapock}

\section{Les Karipuna. Une histoire}

L'histoire des Karipuna est indissociable de la délimitation de la frontière franco-brésilienne sur le fleuve Oyapock en 1900 et de I'histoire de ce que I'on a appelé le "Territoire Contesté ", devenu aujourd'hui I'État brésilien d'Amapá, longtemps réclamé à la fois par la France et par le Portugal, puis par le Brésil. Échappant de fait à toute autorité politique, loin des centres économiques du Brésil et difficilement accessible depuis la grande ville brésilienne la plus proche, Belém, ce territoire a longtemps représenté un refuge pour les Amérindiens chassés par la pression portugaise, pour les esclaves noirs fugitifs (brésiliens ou guyanais) et plus tard pour les populations pauvres du bas Amazone subissant la répression de la révolte que I'on a appelée le "Cabanagem ", au milieu des années $1830^{4}$. Le "Territoire Contesté " a toujours eu des liens privilégiés avec la Guyane française, vers laquelle s'orientaient jusqu'au début du XXe siècle la plus grande partie des flux économiques (produits de l'élevage, de l'agriculture, de la pêche) et des déplacements. Par ailleurs, à partir de la fin du XIXe siècle, I'exploitation de l'or, mais aussi du balata et du " bois de rose $\|^{5}$, avait attiré vers le "Contesté " des Européens et des Créoles venus de Guyane ou des Antilles françaises et anglaises.

Les Karipuna actuels ${ }^{6}$ se sont formés à partir de ces populations venues du bas Amazone, du Pará ou de Guyane, qui se sont fondues ou qui ont cohabité avec les populations indigènes installées antérieurement (Grenand, 1987) pour

\footnotetext{
4 Cette révolte du "Cabanagem " a mobilisé entre 1835 et 1840 les Noirs libres, les Métis, les Amérindiens détribalisés du bas Amazone contre le gouvernement central et les propriétaires blancs; elle fut très brutalement réprimée.

5 Le tronc du balata produit un latex utilisé dans l'industrie, et le " bois de rose " donne par distillation une huile essentielle destinée à la parfumerie.

6 D’autres populations amérindiennes sont présentes dans cette région : les GalibiMarworno installés sur la rivière Uaçá, dont l'histoire et la situation actuelle sont très proches de celles des Karipuna, et les Palikur, qui ont conservé leur langue et les traits principaux de leur organisation sociale et de leur culture. Un petit village rassemble aussi quelques Kali'na, issus d'une migration familiale depuis l'ouest de la Guyane dans les années 1950.
} 
constituer, en un peu plus d'un siècle, une culture et une société originales (Tassinari, 2002). Ceux que l'on appelait encore au début du XXe siècle les "Brésiliens du Curipi " (du nom de la rivière sur laquelle ils sont installés) alors quelque 150 personnes seulement - devinrent "Indiens " aux yeux de l'administration dans les années 1930, lorsque le Brésil décida de mettre sous la tutelle du Serviço de Proteção aos Índios (SPI) ${ }^{7}$ les indigènes de l'ancien "Contesté ", depuis longtemps tournés vers la Guyane française. Les villages furent alors ouverts à la " brasilianisation " à travers l'éducation scolaire, la généralisation de l'apprentissage du portugais et l'inculcation des ferments nationalistes. La population karipuna, environ 2400 personnes recensées en $2010^{8}$, est aujourd'hui établie dans trois villages principaux sur le Curipi - Manga, Santa Isabel, Espirito Santo - et dans quelques autres petits villages sur la rivière ou déplacés près du tracé de la route menant vers Macapá (BR 156). Avec les Palikur, les Kali'na et les Galibi-Marworno, les Karipuna occupent des territoires indigènes qui leur ont été attribués en 1991, dont la gestion est assurée collectivement (Tassinari, 2002 ; Vidal, 1999 et 2007).

\section{Carte 1 : Les principaux villages karipuna sur la rivière Curipi}
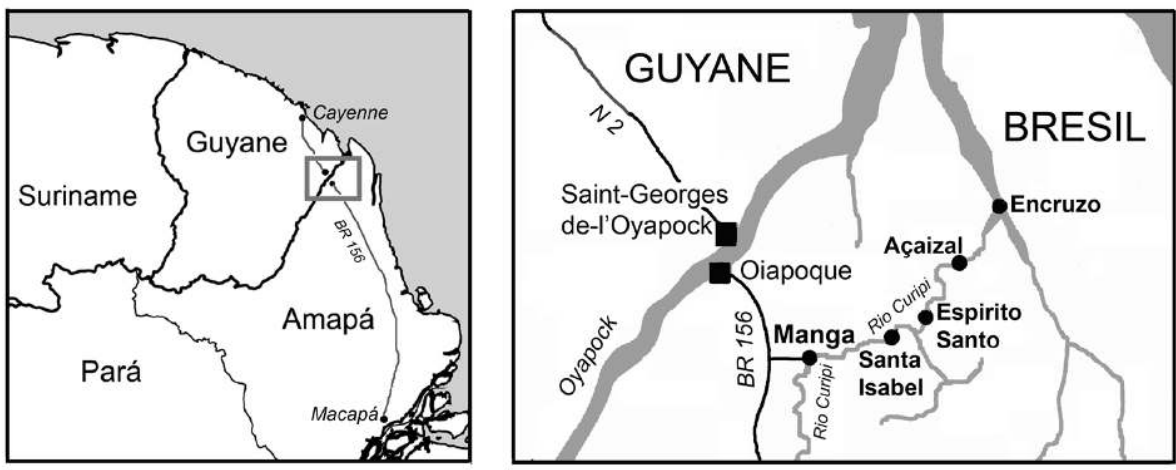

Si les Karipuna se considèrent aujourd'hui eux-mêmes comme "Indiens ", ils ne parlent pas une langue amérindienne mais une variante du créole guyanais, appelée localement patua, qui est devenue réellement ici une langue ethnique opposable aux locuteurs lusophones brésiliens. Reconnu comme langue indigène par le Brésil, le patua fait à ce titre depuis plusieurs années l'objet d'un enseignement spécifique dans les écoles des villages par des "professeurs indigènes ". L'adoption du créole guyanais par les Karipuna ${ }^{9}$, comme langue véhiculaire sans doute dans un premier temps puis comme langue vernaculaire,

7 Le SPI deviendra en 1968 la FUNAI (Fundação Nacional do Índio), aujourd'hui en charge de l'administration des populations indigènes et des territoires qui leur sont attribués.

8 Selon la FUNASA, citée par I'Instituto Socioambiental (http://pib.socioambiental.org/en/ povo/karipuna-do-amapa).

9 Mais aussi par les Galibi-Marworno installés sur la rivière Uaçá, qui ont connu une histoire comparable. Le patua est aussi aujourd'hui, en complément du portugais, la langue véhiculaire dans les relations avec les Palikur, en particulier dans les assemblées politiques indigènes. II faut noter que cet usage du patua permet aussi aux Karipuna de communiquer et de partager une culture commune avec les populations créolophones de Saint-Georges et des villages du bas Oyapock. 
montre l'importance de l'influence française dans cette zone et témoigne de la densité des rapports qui se sont établis dans I'histoire entre la Guyane et les populations installées sur la rive orientale de la rivière Oyapock.

\section{" Lorsque l'on était Indien, on n'avait pas de problème ! "}

Amorcé au début du XVIle siècle, le peuplement colonial de cette petite région a connu des fortunes diverses jusqu'au moment où, en 1853, fut installé le petit pénitencier de l'Oyapock, qui participait du dispositif de la déportation de forçats vers la Guyane. Après sa fermeture en 1860, le bourg de Saint-Georges de-l'Oyapock demeura un petit centre administratif et commerçant, attirant les populations indigènes et les agriculteurs créoles établis sur les deux rives du fleuve, mais formant aussi la base arrière commerciale des populations venues vers la fin du siècle pour l'exploitation de l'or et du bois de rose dans les rivières du "Contesté ". Saint-Georges s'est d'abord progressivement vidé de sa population, mais a connu ces dernières années une croissance démographique pour atteindre 3600 habitants en 2007 (INSEE). Sur la rive opposée, la création en 1907 d'un poste militaire brésilien destiné à contrôler la nouvelle frontière et d'une petite colonie agricole a permis la formation progressive du petit bourg d'Oiapoque, qui s'est développé rapidement ces dernières décennies pour atteindre aujourd'hui plus de 20000 habitants $^{10}$, en attirant une population brésilienne pauvre en quête de passage vers les villes de Guyane ou vers les chantiers aurifères de l'intérieur.

Jusqu'aux années 2000, avant l'ouverture des routes menant vers Macapá et vers Cayenne, Oiapoque et Saint-Georges n'étaient reliés au reste de l'Amapá ou de la Guyane que par des liaisons aériennes ou fluviales peu nombreuses, une situation qui limitait considérablement la circulation et isolait cette petite région. Ensemble, les deux bourgs et leurs écarts formaient un " pays " ancré de part et $d^{\prime}$ autre d'une frontière politique qui restait toute théorique aux yeux des habitants, deux pôles entre lesquels se déplaçaient les personnes, motivées par la visite aux familles, la recherche d'un travail ou le commerce, sur fond de différentiel économique entre la Guyane, département français, et une région pauvre et quelque peu délaissée du nord brésilien. La route permet aujourd'hui de relier les villages karipuna du Curipi à Oiapoque en une heure, on vient vendre son couac $^{11}$ et ses bananes, acheter dans les boutiques, effectuer des formalités administratives auprès des services de l'État et de la FUNAI, consulter les services de santé. Pour les gens du Curipi, Oiapoque est plus qu'un centre administratif et commercial, c'est véritablement une extension des villages, où I'on peut s'approvisionner, mais aussi retrouver les amis et les parents installés sur place.

Dans les dernières décennies, quelques familles Karipuna se sont installées en face, sur la rive française, près du petit village créole d'Ouanary ou à proximité de Saint-Georges, mais la plupart ne venaient que pour vendre leurs produits agricoles à Oiapoque ou à Saint-Georges, notamment un couac apprécié des consommateurs dont la demande est depuis longtemps soutenue par la

10 Selon I'Instituto Brasileiro de Geografia e Estatística (IBGE).

11 Le couac est une farine de manioc torréfiée, de longue conservation, qui constitue la base alimentaire dans toute cette région. 
présence d'une main-d'œuvre nombreuse sur les chantiers d'orpaillage de l'intérieur. Dans les années 1980 des observateurs notaient que "le commerce avec les villes de Guyane française par ceux que I'on appelle les "Indiens brésiliens" a un poids important dans l'économie des Palikur, Galibi et Karipuna " (Ricardo et Gallois, 1983 : 5). Aujourd'hui, quelques Karipuna profitent des opportunités d'emploi sur les chantiers des travaux d'infrastructures, récemment relancés par la construction du pont qui doit relier les deux rives. D'autres, plus nombreux, continuent de venir vendre leur production agricole au marché ou directement à des habitants qui sont leurs clients réguliers. À Saint-Georges, comme à Oiapoque, les familles qui se sont installées ${ }^{12}$ représentent autant de points $\mathrm{d}^{\prime}$ accueil potentiels et de relais sociaux ou commerciaux entre les villages du Curipi et Cayenne.

Les plus anciens parmi les Karipuna évoquent aujourd'hui comme une évidence ce petit monde de l'Oyapock à l'intérieur duquel, "lorsque l'on était Indien, on n'avait pas de problème ! ". La proximité des deux bourgs et leur enclavement, la pratique commune de la langue créole et une certaine homogénéité des modes de vie, une histoire partagée, ont longtemps favorisé l'établissement de liens entre les deux rives (" avant, les gens de Saint-Georges, les douaniers, les gendarmes, participaient à la vie des villages, ils venaient à Manga. Ils venaient jouer au foot, nous on allait jouer là-bas "). Et, au fil des années, les stratégies qui permettent de tirer bénéfice du différentiel économique entre la Guyane et le Brésil, mais tout autant la densité des liens sociaux créés dans I'histoire, ont construit des configurations familiales complexes ancrées dans les deux pays. Ce microcosme de l'Oyapock connaît aujourd'hui, pour quelques familles karipuna, des prolongements jusque vers Macapá, la capitale de l'État, où travaillent ou étudient un certain nombre de personnes, mais l'espace social karipuna est beaucoup plus ouvert en direction de la Guyane française, irrigué par les réseaux familiaux et se nourrissant d'un écart économique qui reste encore important.

Si la présence karipuna en Guyane n'a jamais représenté une population importante (sans doute, globalement, moins de 200 personnes simultanément), les flux ont été constants au cours des dernières décennies, prolongeant une histoire qui avait poussé les Karipuna à regarder vers la Guyane plus que vers le Brésil. Le " pays " du bas Oyapock représentait aux yeux des villageois du Curipi un petit monde dont ils avaient le sentiment d'être une des composantes et le départ vers Cayenne était pensé comme un prolongement de ces mobilités entre les deux rives ; il s'inscrivait dans les mêmes logiques. Ainsi que l'exprimaient sur un mode plaisant ces Karipuna croisés dans le bourg d'Oiapoque : " a terra entre o Brasil e Cayenne é nossa! " ${ }^{13}$.

12 Le " capitaine " du village amérindien Palikur estime à quatre-vingts le nombre de Karipuna installés à Saint-Georges et à cinquante les Galibi-Marworno, de leur nom créole moun Uaçá (litt. " les gens de la rivière Uaçá »). Un certain nombre de femmes sont mariées sur place avec des Guyanais.

13 " La terre entre le Brésil et Cayenne est à nous ! ". 


\section{"Aller en France »}

Les familles karipuna rencontrées à Cayenne dans le cadre de l'enquête sont pour la plupart originaires de Manga, le plus gros des villages du Curipi. Dans ce village, qui connaît aujourd'hui une petite prospérité, mais toute relative et récente, les gens se souviennent que, dans un passé pas très éloigné, beaucoup d'hommes voulaient échapper à ce qu'ils considéraient comme une situation misérable, marquée par une économie de quasi subsistance que ne suffisaient pas à compenser la vente des productions agricoles ou les petits emplois occupés occasionnellement à Saint-Georges ou à Oiapoque. Les jeunes surtout, et plus encore lorsqu'ils n'avaient pas de responsabilité familiale, rêvaient de venir travailler " en France ", c'est-à-dire, pour la plupart, à Cayenne, plus proche et plus accessible que les villes brésiliennes de Macapá ou de Belém, et offrant surtout des perspectives économiques plus attrayantes.

Ce désir de partir en France semble à cette époque avoir été assez fort et assez partagé dans les villages karipuna - et les départs eux-mêmes assez nombreux - pour que le SPI puis la FUNAI se soient inquiétés dans les années 1970 et 1980 de ce que les responsables appelaient alors " a evasão dos Indios " ${ }^{14}$ vers la Guyane (Ricardo et Gallois, 1983 : 5). Le risque de voir se dépeupler les villages avait alors conduit la FUNAl à tenter de fixer les gens en exerçant un contrôle administratif, en leur apportant une assistance et quelques avantages, et en engageant le processus qui allait conduire à la reconnaissance des " terres indigènes " dans les années 1990.

Mais si la FUNAl pouvait mettre quelques obstacles administratifs au départ (" ils contrôlaient les départs, on ne pouvait pas sortir comme maintenant, il fallait revenir ! "), les anciens migrants s'accordent à reconnaître que partir travailler à Cayenne "sans les papiers " ne soulevait pas de difficulté particulière : "Autrefois, on n'avait pas de problème de papiers, on venait nous chercher jusqu'ici pour travailler et le patron donnait une chambre pour habiter ", "il suffisait de montrer ton certificat de naissance, on voyait que tu étais Indien ". Comme les autres migrants brésiliens en situation irrégulière, beaucoup se retrouvaient dans la situation de fournir une main-d'œuvre bon marché, exploitée, mais même dans ces conditions le départ est resté longtemps avantageux : I'écart des salaires entre la Guyane française et le Brésil pour un même métier était d'un à cinq environ dans les années 1980, et jusqu'à un à douze au plus fort de la crise économique brésilienne de la fin des années 1990 (Arouck, 2000).

Au cours des dernières décennies, la quête d'un travail sur Cayenne par les Karipuna n'a guère été différente, dans ses motivations et dans sa forme, de l'aventure qu'ont vécue à la même époque d'autres Brésiliens, beaucoup plus nombreux, qui venaient de Macapá et de Belém ou des zones rurales du

14 « La fuite des Indiens». 
Nordeste ${ }^{15}$. Ces Brésiliens arrivaient à la frontière en attente d'un passage vers la Guyane, où les hommes pouvaient espérer trouver un emploi dans le bâtiment et les femmes comme employées de maison (Pinto, 2011). Comme eux, les Karipuna qui viennent chercher un travail en Guyane sont des ruraux amazoniens peu qualifiés, possédant généralement un faible niveau d'éducation scolaire et exerçant dans leur ensemble les mêmes métiers dans les secteurs du bâtiment ou des services. Les travailleurs karipuna visaient le plus souvent Cayenne et les bourgs du littoral et ceux qui ont travaillé quelque temps au garimpo ${ }^{16}$ prennent soin de préciser qu'il s'agissait de chantiers dirigés par des "patrons français ", soulignant ainsi la différence avec les garimpos clandestins de la grande forêt, où les conditions de vie et de travail sont beaucoup plus dures - même si des passerelles existent entre les deux univers (Pinto, 2011). À Cayenne, ils se sont installés dans quelques quartiers périphériques rassemblant une forte population brésilienne, majoritairement masculine jusqu'aux années 1990, enchaînant des emplois précaires pour parfois déboucher sur un contrat pérenne lorsque, avec le temps, ils ont pu obtenir "les papiers ${ }^{17}$.

Jusqu'à l'ouverture de la route reliant Cayenne à Saint-Georges, en 2003, les Karipuna qui travaillaient en Guyane avaient moins de contacts avec les villages d'origine qu'aujourd'hui, mais les liens n'étaient jamais longtemps coupés. Un certain nombre ont fait le choix de s'installer durablement en famille à Cayenne, mais la plupart, souvent des hommes jeunes, célibataires, ont enchaîné au fil des années des " jobs ${ }^{18}$, entrecoupés de longs séjours au village, pour revenir s'y établir définitivement lorsque les conditions d'entrée en Guyane sont devenues plus difficiles.

Leur histoire, aux marges du Brésil et aux marges de la Guyane, permettait aux Karipuna de penser leur départ d'une manière bien différente des autres migrants brésiliens. Lorsqu'ils quittaient le Curipi pour venir en Guyane, ils ne

15 Les Karipuna n'ont guère été concernés par le chantier de la construction de la base de Kourou, qui avait attiré entre 1965 et 1970 un nombre important de travailleurs brésiliens recrutés sur contrat. Mais ils ont participé au flux de migration qui s'est installé après la fin du chantier, qui s'est amplifié au début des années 1990, alors que l'activité de l'orpaillage reprenait, attirant des milliers de garimpeiros qui délaissaient des chantiers brésiliens dont la production se tarissait pour rejoindre des rivières plus prometteuses en Guyane. La présence brésilienne s'est ainsi considérablement accrue au cours des dernières décennies, passant de quelque 1500 personnes en 1974 à plus de 20000 aujourd'hui - un chiffre vraisemblablement sous-estimé, tant est importante la présence de migrants illégaux, notamment dans les chantiers d'orpaillage en forêt (Arouck, 2001 ; Pinto, 2011).

16 Le terme brésilien garimpo désigne un petit chantier d'exploitation de l'or, généralement éloigné en forêt et le plus souvent illégal. Rappelons que la découverte de l'or alluvial en 1854 avait provoqué une véritable ruée vers la Guyane (surtout en provenance des petites Antilles), ouvrant un premier cycle d'exploitation qui s'est prolongé, en déclinant, jusque vers 1950. Le cycle actuel, accompagné d'une deuxième ruée, venue du Brésil, a commencé dans les années 1990.

17 La migration brésilienne s'est un peu féminisée ces dernières années, du fait du regroupement familial, ou par la venue de femmes décidant de tenter l'aventure à la recherche d'un emploi domestique, mais acceptant parfois aussi, à l'occasion, ce que Tabet (2004) appelle des " échanges de type économico-sexuel ". Ce qui n'exclut nullement que les mêmes personnes puissent être aussi en quête, à travers ces échanges, de la final feliz (" fin heureuse ") que représenterait un mariage avec un Guyanais ou un métropolitain, permettant à la fois stabilisation économique et ascension sociale (Pinto, $2011: 114)$.

18 Le terme désigne, en Guyane, de petits emplois temporaires, à la tâche, non déclarés. 
partaient pas vers une terre inconnue comme lorsque I'on vient de Macapá, de Belém ou des régions du Nordeste, ils pouvaient enchaîner des séjours à Cayenne et retours au village, dans lequel la plupart sont revenus vivre. Ils s'inscrivaient aussi, dans une certaine mesure, dans une continuité historique et culturelle entre l'est de la Guyane et le nord de l'Amapá, que soulignait par exemple l'utilisation commune de la langue créole par les Karipuna et les Guyanais, une langue partagée qui facilitait le premier contact et l'échange professionnel, et autorisait une certaine connivence créatrice de lien social : "Dans ma famille, on parlait toujours le patua, c'est presque la même chose que le créole. Pour nous, c'était plus facile que pour les Brésiliens qui arrivent de Belém par exemple, qui ne parlent ni patua ni français " $"$.

Et surtout, lorsqu'ils tentaient l'aventure de venir à Cayenne, les Karipuna s'appuyaient bien souvent sur des réseaux familiaux ou villageois déjà établis entre les villages de départ et les divers points d'installation en Guyane, des réseaux au sein desquels pouvaient s'organiser la solidarité et qui étaient susceptibles d'être mobilisés pour faciliter le voyage, l'installation ou l'insertion professionnelle.

\section{Le village dans la ville}

\section{L'exemple des familles karipuna de la Bp.134}

Un certain nombre de familles Karipuna établies à Cayenne habitent ce que I'on appelle " la Bp.134 ", un quartier brésilien situé dans la périphérie de la ville, sur la commune de Remire-Montjoly ${ }^{20}$. Lors de la formation du quartier, dans les années 1990, la mairie avait chargé un des premiers occupants, João, de gérer l'arrivée des familles et d'accorder une parcelle à celles dont la demande était acceptée. João, lui-même " brésilien " originaire de Macapá, est marié à une "Indienne " de Manga. II a tout naturellement organisé l'installation de familles karipuna dans le secteur dans lequel il s'était établi. En 2011, dans cette partie du quartier, huit unités résidentielles abritent une trentaine d'adultes karipuna, familles nucléaires ou familles étendues, couples karipuna ou couples mixtes. Pour la plupart, ces personnes sont en situation régulière (mais pas toutes), ce qui leur permet de gérer plus facilement leur rapport au village et les allersretours vers le Brésil. Ce sont toutes des familles originaires de Manga, souvent installées de longue date, qui ont entre elles de multiples liens de consanguinité ou d'affinité et qui développent les mêmes formes de sociabilité qu'au village : si les gens n'ont ordinairement que peu de contacts au quotidien hors de l'unité

\footnotetext{
19 L'avantage conféré par la pratique du patua est toutefois allé en s'amenuisant car la connaissance de cette langue s'est affaiblie au village au profit de pratiques bilingues dans lesquelles le portugais domine de plus en plus, notamment pour les plus jeunes générations (Musolino, 2000), mais aussi du fait de la place plus importante occupée par le français dans les échanges en Guyane.

20 Ce quartier, situé sur la commune de Remire-Montjoly est issu de la réinstallation de 122 familles brésiliennes précédemment installées vers Fort Diamant, sur la route des plages en 1992. La population s'est regroupée sur la base des réseaux familiaux. $90 \%$ des familles sont originaires de Macapá, $10 \%$ de Belém et d'Oiapoque. La population est estimée à 1 500-1 700 personnes selon I'INSEE (2007), et 3 000-3 500 selon le responsable de l'association de quartier [Piantoni, 2009b]. La Bp.134 est devenue officiellement en 2008 la Résidence "Arc-en-ciel ", mais I'usage a généralement maintenu I'ancienne appellation au sein même du village.
} 
résidentielle, et cela d'autant moins que les occasions de travail collectif sont ici beaucoup plus rares que dans les villages du Curipi, ils déploient par contre une sociabilité élargie à l'occasion des fêtes familiales et des fêtes calendaires.

En remontant les réseaux sociaux reliant les familles de la Bp.134 au village de Manga, on observe que la présence karipuna en Guyane prend la forme de constellations familiales qui rassemblent des individus et des ménages établis à Cayenne, à Saint-Georges et dans les villages du Curipi, ce qui constitue sans doute une des spécificités de cette présence karipuna en regard de la situation des autres migrants brésiliens. Ces configurations font apparaître tout à la fois les nombreux liens familiaux qui unissent les migrants entre eux et (pour les plus jeunes générations) les alliances réalisées avec des non Karipuna, en particulier à travers les mariages avec des hommes créoles guyanais ou " métropolitains ". Ouvertes à la fois sur les villages du Curipi et sur leur environnement social guyanais, ces constellations familiales forment ainsi des réseaux à l'intérieur desquels se diffusent les informations sur les possibilités de départ vers la Guyane, et circulent les personnes, qui peuvent y trouver des points d'accueil. $C^{\prime}$ est aussi cette forme d'organisation et d'articulation des familles qui soutient les petites activités commerciales (en particulier la revente à Cayenne du couac produit au village par la famille) auxquelles se livrent nombre de Karipuna installés à Cayenne, ce qui contribue à tisser ou à resserrer les liens avec les villages.

Plus généralement, la présence d'un membre de la famille déjà établi à Cayenne facilite l'arrivée et l'installation, en équilibrant les situations de ceux qui ont pu obtenir une carte de séjour, et de ceux qui arrivent sans visa et restent dans une illégalité aujourd'hui beaucoup plus pesante que par le passé en raison de la pression exercée par les contrôles des personnes et l'augmentation des reconduites à la frontière. Enfin, au moins jusqu'à ces dernières années, cette présence permettait dans bien des cas l'insertion professionnelle des nouveaux arrivants. Des configurations familiales, à la fois professionnelles et spatiales, sont ainsi identifiables, comme par exemple autour d'une petite entreprise d'embouteillage de soda en périphérie de Cayenne, dans laquelle plusieurs hommes de Manga ont successivement ou simultanément travaillé ; elle emploie aujourd'hui deux Karipuna qui logent leur famille dans une maison construite à l'arrière de l'usine, sur un terrain accordé par le " patron ".

\section{« Revenir au village " Manga comme refuge et comme fabrique d'identité}

Par le passé, alors que les conditions de transport étaient plus difficiles, ceux qui venaient travailler en Guyane avaient des contacts moins fréquents avec le village, mais la plupart enchaînaient emplois temporaires et séjours au village, et beaucoup revenaient s'installer définitivement à Manga. Un certain nombre de ces migrants ont aujourd'hui fait le choix de s'établir en Guyane en famille, mais les liens que ces Karipuna de Cayenne ont toujours maintenus avec les villages se sont encore renforcés avec la facilité de déplacement apportée par la route de l'est de la Guyane, permettant aux personnes disposant " des papiers " de réaliser un aller et retour dans la journée. Lieu de résidence pour les vacances ou pour la retraite pour certaines des familles de la Bp.134, les villages représentent aussi une base de repli, où I'on a son assise familiale, un logement assuré, et 
où l'on sait qu'il serait possible de vivre en pratiquant l'agriculture si la situation se faisait difficile en Guyane. Les gens sont désormais en contact par téléphone avec la famille (" quand il y a quelqu'un de malade on est prévenu tout de suite, et on peut partir pour passer quelques jours à Manga "). On accueille les parents qui souhaitent venir en Guyane pour recevoir des soins, et comme les autres migrants, on s'attache lorsqu'on le peut à aider financièrement les membres de la famille restés au village.

Cette permanence de la relation avec les villages du Curipi se laisse lire aujourd'hui, par exemple, dans les choix matrimoniaux : si un certain nombre de jeunes femmes karipuna de Cayenne se sont mariées avec un Guyanais ou un métropolitain, une majorité de jeunes hommes fait le choix d'un mariage au village. Ils suivent en cela les logiques matrimoniales mises en œuvre par les villageois karipuna du Curipi, qui privilégient les alliances avec le " très proche ", ce qui a permis à la société karipuna de se construire depuis le début du XIXe siècle en tissant de multiples liens entre les familles des villages - qui étaient par ailleurs prises dans un réseau serré de liens de consanguinité du fait de la petite taille des groupes (Tassinari, 2002). Mais ces choix révèlent aussi des stratégies d'hypergamie de la part de ces familles villageoises qui valorisent les unions avec un Karipuna établi en Guyane (" À Manga, beaucoup de mères rêvent de voir leur fille mariée en Guyane "), réalisant ainsi une forme de mobilité sociale ascendante et renforçant leur lien économique avec les migrants.

Pour ces familles établies en Guyane dans les " quartiers " brésiliens, Manga et les autres villages sont aussi des lieux où l'on peut se replonger dans un espace villageois, où I'on peut véritablement se vivre comme membre d'une communauté. C'est en particulier le cas lors des fêtes de fin d'années ou lors des grandes fêtes religieuses qui rythment l'année sur le Curipi, auxquelles on $s^{\prime}$ efforce de participer, ou dans l'organisation desquelles certains s'engagent ${ }^{21}$. Pour ceux qui sont à Cayenne, c'est l'occasion d'un retour dans la famille mais surtout d'une implication dans la sociabilité particulière générée par ces fêtes, qui sont avant tout dans les villages des moments au cours desquels la communauté se rassemble et à travers lesquels se " construit " le monde karipuna (Tassinari, 2002).

$C^{\prime}$ est dans cette proximité avec le village, dans la participation à une sociabilité villageoise produisant du collectif, que l'on peut lire les logiques à travers lesquelles les gens du Curipi ont longtemps pensé leur présence à Cayenne ou plus généralement en Guyane. Mais, depuis quelques années ces logiques sont confrontées à des changements qui ont modifié les conditions de venue en Guyane, et qui ont aussi partiellement reconfiguré le contexte villageois de départ. Ces changements marquent une double coupure et fragilisent la représentation ancienne largement partagée d'une sorte de continuum géographique et social au sein duquel les Karipuna inscrivaient leurs mobilités.

21 Les fêtes des " saints patrons " des villages mettent en jeu une promesse faite au Saint-Esprit ou à un Saint par des personnes qui sollicitent une intercession, et qui en retour décident d'en prendre en charge l'organisation. La préparation et le déroulement de ces fêtes sont conduits à travers des formes de travail collectif pour lesquels les familles mobilisent de multiples réseaux d'échange et d'appui, formalisés aux différents niveaux d'organisation de la société karipuna : famille étendue, groupes locaux, communautés de villages. 


\section{Une double coupure}

\section{La « frontière "}

La route vers Saint-Georges de-l'Oyapock, qui facilite les mouvements vers et depuis Cayenne, a été ouverte au moment où la France engageait un renforcement du contrôle de l'arrivée des migrants en Guyane. À la même époque, et pour les mêmes raisons, l'obtention d'un visa ou, dans un second temps, d'un titre de séjour devenait plus difficile pour ces migrants ${ }^{22}$. La première coupure à laquelle ont été confrontés les Karipuna a ainsi été celle d'une frontière qui s'est révélée soudain plus réelle, moins sur le strict plan de la circulation entre Saint-Georges et Cayenne (le barrage de police qui contrôle la route est, en pratique, contournable) que par la pression qu'elle imposait au quotidien. Cette pression se fait sentir à Cayenne à travers des contrôles policiers plus nombreux et plus stricts, notamment sur les lieux de travail, mais elle s'exerce aussi à Saint-Georges, marquant brutalement la frontière, là où elle n'avait en quelque sorte jamais véritablement existé. La vie dans ce petit monde de l'Oiapoque s'est ainsi brusquement transformée pour les Karipuna qui avaient I'habitude, depuis longtemps, de venir proposer leurs produits agricoles dans le bourg français.

À la fin de l'année 2008, un incident a marqué les esprits, que l'on relatait encore plus d'un an après pour expliquer à quel point les choses avaient changé : ce jour-là, la Police aux frontières avait jeté à la rivière tout le couac et les autres produits que vendaient les Karipuna et les Galibi-Marworno venus de la rive brésilienne, et avaient interpellé un des vendeurs qui n'avait été libéré, après plusieurs heures, que grâce à l'intervention des responsables de la FUNAI d'Oiapoque. Cet incident avait suscité une grande émotion dans les villages, ainsi que me l'expliquait le cacique de Manga qui voyait en moi un possible représentant de l'administration française. Les habitants ont alors eu le sentiment d'être confrontés à des mesures injustes et vexatoires, d'autant plus qu'ils constataient la facilité avec laquelle les Français pouvaient venir à Oiapoque en touristes. Depuis lors, la situation a changé, la position de la police s'est un peu assouplie vis-à-vis des vendeurs karipuna, mais surtout la lente valorisation du real brésilien face à l'euro a rendu la vente à Saint-Georges moins intéressante. Et si aujourd'hui il reste toujours possible pour quelques-uns munis " des papiers" de traverser l'Oyapock pour venir travailler sur les chantiers de Saint-Georges ou dans un commerce, l'imaginaire karipuna qui projetait une histoire et construisait un réseau social dans un espace pensé comme transfrontalier est désormais fragilisé et remis en question.

\section{Manga}

Mais le changement n'est pas intervenu seulement sur la frontière, il a progressivement touché les villages du Curipi qui ne présentent plus le même profil qu'évoquaient les " vieux " migrants pour justifier leur désir de départ. À

\footnotetext{
$22 \mathrm{La}$ " route nationale $\mathrm{n}^{\circ} 2$ ", ouverte à la circulation dès 2003, a été terminée en 2005 , et le durcissement des politiques de contrôle des migrations s'est fait sentir à partir de 2006. Cette année-là le nombre de reconduites à la frontière de Brésiliens et de Surinamais a presque doublé, atteignant 4036 personnes pour les seuls Brésiliens, soit I'effectif le plus important des reconduites. II faut noter que ce chiffre avait été multiplié par douze depuis 1996 (Granger, 2008).
} 
Manga, la vie demeure difficile pour la plupart des habitants, mais ce n'est plus ce village dont on espérait naguère pouvoir s'évader en venant travailler en Guyane. Si les niveaux de vie restent globalement modestes, le revenu généré par l'agriculture est désormais significatif, et pour la plupart des ménages la faiblesse du revenu monétaire est compensée par la permanence des échanges de services, de savoir-faire et de biens, et par les systèmes d'entraide. Et la présence d'une petite population de fonctionnaires (il y a ainsi par exemple aujourd'hui à Manga seize enseignants venus de l'extérieur), comme le voisinage de la petite ville d'Oiapoque, créent un volant de " jobs " et quelques emplois domestiques.

Le retour et la réinstallation des migrants qui ont effectué plusieurs séjours en Guyane au cours de décennies précédentes n'ont pas modifié en profondeur l'aspect ou la structure socio-économique des villages. Pour la plupart, leurs séjours à Cayenne ne leur ont pas permis de se constituer un petit capital qui aurait, par exemple, pu être investi dans la construction d'une maison " d'émigré ": "Quand tu reviens au village, les gens te croient riche, mais ce n'est pas vrai ! Même si tu as un peu d'argent, tu restes quand même pauvre ! $\grave{A}$ Cayenne, quand on est jeune, on sort, et il y a beaucoup de choses à payer ". Les quelques " belles maisons " à Manga aujourd'hui sont plutôt celles des Karipuna installés définitivement en Guyane, qui ont un emploi depuis longtemps et investissent petit à petit dans le village.

Manga a changé ces dernières années, et surtout le Brésil lui-même s'est transformé, la reprise économique que connaît le pays a rendu moins intéressant le différentiel avec la Guyane et la migration devient moins attractive pour les Karipuna, alors qu'elle le reste dans les régions rurales du Nordeste ${ }^{23}$. Malgré tout, certains seraient encore tentés de partir pour Cayenne, mais ils savent que c'est difficile : "Ils voient qu'ils ne peuvent même plus venir vendre leur couac à Saint-Georges! Ce n'est pas la peine de venir si c'est pour rester cachés! Mais il $y$ a tout de même des gens qui veulent venir en Guyane. Moi je suis arrivé alors que c'était facile!".

À l'instar de la plupart des migrants brésiliens, les Karipuna de Cayenne ont longtemps pensé leur présence en Guyane comme un " entre-deux ", un passage et une attente avant un retour souhaité au village (Arouck, 2001). Aujourd'hui un certain nombre des personnes qui avaient migré au cours des années précédentes se sont progressivement fixées, en famille, lorsqu'elles avaient pu régulariser administrativement leur situation. L'avantage d'un travail régulier et l'attrait du système de protection sociale français, les liens sociaux tissés, la scolarisation des enfants nés sur place et souvent leur choix de vivre en Guyane, ont constitué de fortes incitations à s'établir durablement. Pour ces familles, le mouvement qui portait les villageois du Curipi vers la Guyane s'est d'une certaine manière inversé et elles sont amenées aujourd'hui à établir un nouveau rapport au village, désormais objet d'autres investissements symboliques et d'autres formes de mobilités, cette fois depuis la ville.

23 Depuis le début des années 2000, le nombre des migrants originaires de cette région qui arrivent à Oiapoque, et singulièrement ceux venant du Maranhão, est en forte augmentation (Pinto, 2011). 


\section{Se découvrir brésilien}

Qu'ils arrivent aujourd'hui en Guyane, ou qu'ils y résident depuis plusieurs années, les Karipuna de la Bp.134 parlent portugais entre eux au quotidien plutôt que le patua, ils regardent la télévision brésilienne et ils sont installés dans un quartier peuplé majoritairement d'autres migrants venus du Brésil. Dans les villages du Curipi, ils ont obtenu la reconnaissance d'une identité amérindienne à laquelle ils ont pu donner une forme sociale et politique, sur une terre indigène qui leur a été attribuée. Et pendant longtemps ils ont pu se déplacer vers Cayenne sans que leur soit posée la question de leur origine, dans cet espace qui formait pour eux une sorte de prolongement du petit monde de l'Oyapock. Mais désormais, aux yeux des autorités, de l'administration et de la population de la Guyane, les Karipuna ne sont plus ces "Indiens " dont on facilitait la venue mais dont on a désormais oublié jusqu'à l'existence, ils sont devenus des " migrants brésiliens " dont on s'efforce de contrôler l'arrivée.

"Avant, il n'y avait pas de problème, on pouvait aller et venir. Maintenant c'est différent, il y a beaucoup de Brésiliens, c'est devenu difficile ". La formule, récurrente lors des entretiens, est paradoxale dans la bouche de ces migrants venus du Brésil, elle renvoie à la relation complexe que les Karipuna entretiennent globalement avec ce pays, qu'il faut éclairer en revenant sur leur histoire. S'ils se réfèrent volontiers à leur " indianité ", en référence à un héritage culturel indigène de l'ancien "Contesté " et en regard du statut d'indigène que le Brésil leur reconnaît, ils participent tout autant d'une " brasilianité " des familles fondatrices des villages, qui sont venues du bas Amazone et du Pará au cours du XIXe siècle. Mais plus encore, la société karipuna est aujourd'hui profondément imprégnée d'un sentiment national que l'État brésilien a diffusé dans les villages depuis des décennies, notamment à travers l'éducation scolaire. Ainsi que I'explique Tassinari (1998), ce que I'on peut lire aujourd'hui comme une identité karipuna s'est construite à partir de ce double héritage, c'est " une identité ethnique qui est capable d'englober en elle-même des termes tels que "indien" et "brésilien", sans conférer à aucun de ces deux pôles une antériorité ou une prédominance ", chacune de ces références identitaires s'inscrivant dans le calendrier des fêtes civiles qui rythment l'année dans les villages : " dia do Indio " le 19 avril, " dia da Pátria " le 7 septembre. II n'est donc pas étonnant de voir que les Karipuna établis en Guyane se considèrent eux-mêmes comme des Brésiliens, ils revendiquent cette appartenance citoyenne, et la plupart expriment volontiers le souhait de vivre au Brésil s'ils le pouvaient, plutôt qu'en Guyane, dont ils reconnaissent les avantages mais où ils ne retrouvent pas une " ambiance ", une sociabilité, une manière de vivre qu'ils disent regretter ${ }^{24}$.

Ce n'est donc pas leur "brasilianité " qui leur pose problème, mais plutôt la pression exercée au cours des dernières décennies par un autre Brésil que celui qu'ils avaient jusque-là connu, celui des migrants venus nombreux des villes ou du lointain Nordeste, qui forment en Guyane deux groupes identifiables, au-delà de fortes disparités économiques et sociales reflétant les hiérarchies de la société brésilienne elle-même (Chérubini, 1988 ; Piantoni, 2009a). D'une part, des

$24 C^{\prime}$ est cette même nostalgie du Brésil qu'évoque Police à propos des migrants brésiliens à Cayenne: "Le Brésil leur manque, presque toujours, sauf à ne s'intéresser qu'aux satisfactions matérielles " (Police, $2010: 67)$. 
familles arrivées dans les années 1970 et 1980 à Cayenne et à Kourou, souvent des personnes issues des petites classes moyennes de Belém ou de Macapá, qui sont venues travailler en Guyane avec I'objectif de réinvestir au Brésil dans un commerce ou dans l'immobilier ${ }^{25}$. Ils sont installés de manière pérenne, et généralement en situation administrative régulière, mais constituent un groupe peu nombreux. D'autre part, les gros effectifs d'une migration de subsistance et de transferts familiaux qui s'est développée depuis une vingtaine d'années, alimentée notamment par l'activité de l'orpaillage, une migration qui a conduit vers la Guyane une population très pauvre, souvent issue de régions qui ne ressortissent pas à un espace amazonien dans lequel les Karipuna peuvent se reconnaître.

Les Karipuna de la Bp.134 se pensent - et, de fait, sont - doublement différents de ces Brésiliens qu'ils rencontrent aujourd'hui à Cayenne ou à SaintGeorges, et de la représentation qui leur est associée en Guyane. Si quelquesuns préparent leur retour au village pour la retraite en aménageant une maison, la plupart n'ont pas en eux le rêve d'investir dans un commerce ou une maison locative au pays (ce qui, au demeurant, reste un rêve et une réalité de citadin) et, pour les migrants qui sont de retour à Manga après avoir travaillé en Guyane, l'argent qu'ils ont gagné a irrigué au jour le jour un espace social familial et villageois bien plus qu'il n'a été au cœur d'une accumulation de capital. Mais ils ne se reconnaissent pas non plus dans l'image rude que renvoient ces travailleurs illégaux des quartiers périphériques de Cayenne ou des garimpos, venus plus récemment du Nordeste ou de plus loin, qui nourrissent le stéréotype du Brésilien voleur et querelleur. Ni entrepreneurs citadins, ni sous-prolétariat nordestin, les Karipuna sont néanmoins désormais perçus à travers une grille de lecture ethnicisante et globalisante qui tend aujourd'hui en Guyane à construire une image commune du "Brésilien ", globalement dépréciative, qu'ils partagent malgré eux (Police, 2010), et ils subissent comme les autres migrants brésiliens le durcissement des politiques d'immigration et l'arbitraire qui leur est souvent associé sur le terrain.

Face à cela, le recours aux jeux de l'ethnicité et l'affirmation de leur indianité pourrait apparaître comme une ressource et leur offrir une stratégie alternative efficace, dans une Guyane où le mouvement revendicatif amérindien a pu imposer depuis plusieurs années une certaine reconnaissance du fait autochtone (Collomb, 2001). C'est par exemple de cette manière que les Palikur, originaires de la même région que les Karipuna dans I'arrière-pays d'Oiapoque, ont construit leur présence en Guyane, à Saint-Georges et dans la périphérie de Cayenne. Ils ont eux aussi migré en nombre vers la Guyane au cours des dernières décennies ${ }^{26}$, mais ils peuvent se réclamer d'une ascendance amérindienne incontestable, qui leur est reconnue en Guyane même où leur place dans l'espace politique et culturel indigène n'est pas contestée. Plus nombreux que les Karipuna, ils ont créé de gros villages et ne se sont pas fondus dans les quartiers

25 Dans la Bp.134, à proximité de I'habitat modeste des familles karipuna, on me désigne un gros bâtiment: " c'est la maison d'un artisan brésilien, il habite en bas, et en haut il a construit des chambres qu'il loue..." ".

26 Mais moins à la recherche d'un travail que pour résoudre, par le départ de certaines familles, de graves différents internes aux villages d'origine, situés sur la rivière Urukawa, à l'est du Curipi. 
brésiliens. Ils ont conservé les grands traits d'une culture ancestrale, pratiquent toujours leur langue (Mussolino, 2006 ; Capiberibe, 2009) et échappent de fait à cette identité " brésilienne " assignée qui pèse aujourd'hui sur les Karipuna.

Mais les villageois du Curipi ne sont pas en mesure de mettre en œuvre cette stratégie du recours à l'affirmation ethnique, car la part "indienne " de l'identité karipuna, qui revêtait pour eux un caractère d'évidence lors des anciennes mobilités vers la Guyane, ne va pas de soi en Guyane aujourd'hui ${ }^{27}$ : cette indianité Karipuna, dont on a rappelé plus haut combien elle était atypique dans l'espace régional, parce que récemment recomposée et réappropriée, ne peut trouver une légitimité en Guyane, où la figure de I'Indien s'est construite historiquement à travers un imaginaire qui se nourrit, pour l'essentiel, du stéréotype des sociétés et des cultures indigènes de la grande forêt amazonienne (Collomb, 2008). C'est donc un imaginaire qui peine à se saisir de ces populations " mélangées " du "Contesté ", tels les Karipuna, que les colons et les Créoles de Guyane avaient déjà quelque difficulté à caractériser au début du siècle dernier : "Dans le bas Oyapock vit une population d'environ 600 Indiens vêtus, complètement créolisés, produisant et commerçant pour le moins autant que les autres Créoles, ayant des goélettes pour leurs grandes pêches et leurs voyages à Cayenne et de grands abatis fournissant la bonne partie de la farine de manioc consommée dans la colonie. [...] Ces tribus sont les seules civilisées de toute notre population indienne " (Laporte, 1915).

Ces interrogations et ces inquiétudes qui marquent les Karipuna, remettant en question une histoire et d'une certaine manière une identité collective construite dans cette histoire, sont certes suscitées par le nouvel environnement social, politique, économique auquel ils sont désormais confrontés en Guyane et particulièrement à Cayenne. Mais elles les renvoient aussi à la situation nouvelle créée par l'arrivée récente d'une forte présence "brésilienne " dans la petite région d'Oiapoque où sont installés les villages. Poussés par la nécessité et souvent attirés par les promesses d'une entrée en Guyane, ces migrants sont notamment porteurs d'autres modèles de gestion et d'exploitation de l'espace forestier et ils s'opposent parfois aux "Indiens ${ }^{28}$. Les villageois du Curipi se plaignent ainsi des incursions de ces "Brésiliens " sur leurs terres indigènes et en particulier de la pression croissante exercée sur les ressources par les chasseurs professionnels. Mais ils se voient également reprocher de ne pas exploiter " correctement " - c'est-à-dire par une agriculture intensive ou par l'élevage - des terres qu'ils cultivent traditionnellement par rotation de parcelles défrichées en forêt ("ils disent que nous sommes paresseux ! " déplore-t-on à Manga), et ils sont accusés de bloquer par leurs revendications l'achèvement des travaux de la route vers Macapá, qui traverse les terres indigènes.

27 Cette impossibilité pour les Karipuna à s'affirmer - ou à se faire reconnaître - comme "Indiens " dans l'espace guyanais fait en quelque sorte écho au statut de puguty, c'està-dire d'Indiens " misturados " ou " não-puros ", qui est le leur aux yeux de leurs voisins Palikur (Capiberibe, 2009).

28 Comme c'est souvent le cas au Brésil sur les fronts pionniers, lorsque naissent des conflits d'usage sur les terres indigènes (LeTourneau, 2006). 


\section{Conclusion}

Le malaise qu'expriment aujourd'hui les Karipuna dans le nouveau contexte de leurs déplacements vers la Guyane éclaire d'une manière particulière les circulations migratoires qui prennent place dans l'espace régional, et contribue à nourrir le débat ouvert à partir de la notion de "champ transnational " (Berthomière et Hily, 2006) en inversant en quelque sorte les termes classiquement posés. Si la notion mettait en avant l'idée d'un continuum, dans la migration, entre espace d'origine et espace de départ, dans ce cas c'est bien le développement d'un nouvel espace de circulations migratoires entre Brésil et Guyane qui a contribué à refermer ou segmenter le continuum historique, culturel et social, dans lequel les Karipuna pouvaient se projeter et inscrire des mobilités individuelles et familiales. Dans ces formes de mobilités qu'ils pratiquaient, la frontière était certes une donnée de l'histoire, mais elle n'intervenait nullement pour eux comme un obstacle ; c'était plutôt l'expression d'une variable socio-économique produisant un différentiel entre la Guyane et le Brésil, dont ils tiraient avantage. Aujourd'hui l'irruption des migrants venus d'un Brésil lointain, qui leur est dans une large mesure étranger, a donné corps à une ligne de séparation et à une barrière. Évoquant avec nostalgie le temps où "il suffisait de montrer ton certificat de naissance, on voyait que tu étais Indien ", les Karipuna ont donc désormais à franchir une frontière, ils sont devenus des étrangers, des migrants brésiliens. La situation n'est guère différente sur l'autre frontière de la Guyane, où les populations amérindiennes Kali'na établies traditionnellement à cheval entre la Guyane et le Surinam voisin sont désormais caractérisées, par les villageois eux-mêmes, comme Kali'na " français " et comme Kali'na " surinamiens " - par-delà les multiples liens de parenté et d'alliance qui unissent les familles (Collomb, 2000 et 2008).

La Guyane est sortie il y a quelques décennies d'un statut d'espace postcolonial périphérique délaissé par la France, pour s'inscrire dans un monde globalisé comme une " porte " française et européenne vers l'Amérique du Sud et singulièrement le Brésil, développant encore par là son attractivité vis-à-vis de ses voisins du Sud. Dans ce contexte, l'entrée des sociétés indigènes dans ce monde a eu pour elles des conséquences paradoxales. Si Kali'na et Karipuna pouvaient, par le passé, se penser "Indiens " dans un espace qui s'étendait par-delà la frontière politique, ils sont devenus aujourd'hui des Indiens " français " ou "brésiliens " ou encore "surinamiens ". La globalisation a conduit à une fermeture des frontières et à un plus grand contrôle des flux, et le processus engagé en réponse au développement des migrations brésiliennes les a enfermés plus complètement dans des espaces et des registres sociaux et politiques nationaux.

\section{Références bibliographiques}

Abélès Marc (2008) Anthropologie de la globalisation, Paris, Payot, 280 p.

Arouck Ronaldo de Camargo (2001) Brasileiros na Guiana francesa: fronteiras e construções de alteridades, Belém, Université fédérale du Pará, Núcleo de Altos Estudos Amazônicos, 231 p. 
Arouck Ronaldo de Camargo (2000) Brasileiros na Guiana francesa. Novas migrações internacionais ou exportação de tensões sociais na Amazônia?, Lusotopie, pp. 67-78.

Berthomière William et Hily Marie-Antoinette (2006) Décrire les migrations internationales, Revue Européenne des Migrations Internationales, 22 (2), pp. 67-82.

Capiberibe Artionka (2009) Nas duas margens do rio: alteridade e transformações entre os Palikur na fronteira Brasil/Guiana francesa, Tese de doutorado, Rio de Janeiro, PPGAS/Museu Nacional.

Cherubini Bernard (1988) Cayenne, ville créole et polyethnique, présentation d'André Calmont, Paris et Talence, Karthala et CENADDOM, 262 p.

Collomb Gérard (2008) Chroniques interculturelles en Guyane : un « point de vue " Kali'na, in Gérard Collomb et Marie-José Jolivet Éds., Histoires, identités, logiques ethniques. Amérindiens, Créoles et Noirs marrons en Guyane, Paris, Éditions du CTHS, pp. 45-75.

Collomb Gérard (2001) De l'indien à I'indigène. L'internationalisation des luttes amérindiennes en guyane et les enjeux de l'autochtonie, Recherches Amérindiennes au Québec, 31 (3), pp. 37-47.

Collomb Gérard (2000) Identité et territoire chez les kali'na. À propos d'un récit du retour des morts, Journal de la Société des Américanistes, 86, pp. 147-166.

Glick Schiller Nina, Basch Linda and Blanc-Szanton Cristina (1992) Transnationalism: A new Analytic Framework for Understanding Migration, in Nina Glick Schiller, Linda Basch and Cristina Blanc-Szanton Eds., Towards a Transnational Perspective on Migration: Race, Class, Ethnicity, and Nationalism Reconsidered, New York, Academy of Sciences, pp. 1-24.

Granger Stéphane (2008) La Guyane, un territoire Caraïbe en voie de sud-américanisation, EchoGéo, 6, [en ligne], consulté le 31/07/2012. URL : http://echogeo. revues.org/6503

Grenand Françoise et Grenand Pierre (1987) La côte d'Amapa, de la bouche de I'Amazone à la baie d'Oyapock, à travers la tradition orale Palikur, Boletim do Museu Paraense Emilio Goeldi, Serie Antropologia, 3 (1), pp. 1-77.

Laporte Paul (1983) La Guyane des écoles, Cayenne, Comité guyanais d'Action culturelle A.T.I.P.A. [1915], 185 p.

Le Tourneau François-Michel (2006) Enjeux et conflits autour des territoires amérindiens en Amazonie brésilienne, Problèmes d'Amérique Latine, 60, pp. 71-94.

Musolino Álvaro (2006) Migraçao, identidade e cidadania Palikur na fronteira do Oiapoque e litoral suleste da Guiana francesa, Tese de doutorado, Universidade de Brasília, Centro de Pesquisa e Pós-Graduação sobre as Américas.

Piantoni Frédéric (2009a) L'enjeu migratoire en Guyane française : une géographie politique, Cayenne, lbis rouge, $448 \mathrm{p}$.

Piantoni Frédéric (2009b) Discrimination et fragmentation socio-spatiale dans le bassin d'habitat de Cayenne. Étude sur sept quartiers précarisés, rapport de recherche "Lutte contre les discriminations et promotion de l'égalité des chances: des repères pour I'action ", Cayenne, L'ACSé, Centre de Ressources de la Politique de la ville, $72 \mathrm{p}$. 
Pinto Manoel de Jesus de Souza (2011) O Fetiche do emprego: um estudo sobre as relações de trabalho de brasileiros na Guiana Francesa, São Paulo, Iglu Editoria, 328 p.

Police Gérard (2010) €udorado. Le discours brésilien sur la Guyane française, Cayenne, lbis Rouge, 520 p.

Portes Alejandro (1999) La mondialisation par le bas. L'émergence des communautés transnationales, Actes de la recherche en sciences sociales, 129, pp. 15-25.

Ricardo Carlos Alberto e Gallois Dominique T. (Coords.) (1983) Povos Indígenas no Brasil, vol. 3, Amapá/Norte do Pará, São Paulo, Cedi.

Serges Dorothée (2011) Insertion économique des migrantes brésiliennes en Guyane française, thèse de Sociologie, Université de la Sorbonne nouvelle Paris III, $401 \mathrm{p}$.

Tabet Paola (2004) La grande arnaque. Sexualité des femmes et échange économico-sexuel, Paris, L'Harmattan, Bibliothèque du féminisme, 207 p.

Tassinari Antonella (2002) No bom da festa: o processo de construção cultural das famílias karipuna do Amapá, São Paulo, EDUSP, 413 p.

Tassinari Antonella (1998) 'Karipunas' e 'Brasileiros'. A Trajetória de dois Termos, XXI Encontro Annual da ANPOCS, Caxambu, 27 a 31 de outubro de 1998, multigr.

Vidal Lux Boelitz (2007) Povos Indígenas do Baixo Oiapoque: o éncontro das águas, o encruzo dos saberes e a arte de viver, Rio de Janeiro, IEPÉ - Museu do Índio, $197 \mathrm{p}$.

Vidal Lux Boelitz (1999) O modelo e a marca ou o estilo dos misturados. Cosmologia, História e Estética entre os Povos Indígenas do Uaça, Revista de Antropologia, 42 (1-2), pp. 29-45. 


\section{Gérard Collomb}

\section{... « Indiens " ou « Brésiliens " ? Mobilités karipuna vers Cayenne (Guyane française)}

La Guyane française, entre Surinam et Brésil, reçoit des flux démographiques, économiques, culturels qui traversent les frontières héritées de I'histoire coloniale. Quelques groupes amérindiens s'inscrivent d'une manière particulière dans ce dispositif, pratiquant de longue date et jusqu'à aujourd'hui des déplacements de part et d'autre de la frontière politique. L'exemple des Karipuna établis dans le Brésil frontalier et venant travailler en Guyane illustre la place singulière occupée par ces groupes, mais aussi les effets de l'imposition d'une frontière qui était restée jusqu'alors virtuelle. Ces populations sont alors passées du statut $\mathrm{d}^{\prime}$ Indiens véritablement " trans-frontaliers ", bénéficiant de privilèges d'accès au territoire français, à celui de " migrants brésiliens ", auxquels l'État applique des règles renforcées de limitation des mobilités, et ils endossent malgré eux le stéréotype dépréciatif aujourd'hui appliqué aux autres migrants brésiliens en Guyane.

\section{"Indians" or "Brazilians"? Karipuna Mobilities towards Cayenne (French Guiana)}

French Guiana, between Suriname and Brazil, receives demographic, economic, cultural flows, crossing borders inherited from colonial history. Some Native American groups have a special place in this process, moving since long and up to now, on both sides of the politic border. The example of the Karipuna, settled in neighbouring Brazil and coming for to work in Guyana, illustrates this particular place occupied by these groups, but also the effects of the present imposition of a border that previously had long remained virtual. These populations have then moved from the status of "Indians", with privileges for accessing to French territory, to that of "Brazilian migrants", to which the State applies strengthened rules limiting mobilities, and they are from now on seen through the derogatory stereotype applied today to the other Brazilian migrants in Guyana.

\section{$\cdots$ ¿¿ulndios» o «Brasileños»? Movilidades Karipuna a Cayenne (Guayana Francesa)}

La Guayana Francesa, entre Surinam y Brasil, recibe flujos demográficos, económicos y culturales que cruzan las fronteras heredadas de la historia colonial. Algunos grupos de nativos tienen un lugar especial en este proceso, en continuo movimiento desde hace mucho tiempo y hasta ahora, a ambos lados de la frontera política. El ejemplo de los Karipuna, que viven en el vecino Brasil y vienen para trabajar en Guayana, ilustra este lugar particular ocupado por estos grupos, así como también los efectos de la imposición actual de una frontera que anteriormente había permanecido virtual. Estas poblaciones han cambiado de la condición de Indios realmente "transfronterizos", beneficiando de privilegios de acceso al territorio francés, a la de «inmigrantes brasileños» a quienes el Estado aplica normas reforzadas que limitan la movilidad. En consecuencia, los Karipuna son vistos ahora a través de los estereotipos despectivos que actualmente se aplican a otros inmigrantes brasileños en Guyana. 